\title{
Die Diskussion um die Kompetenzverteilung bei der Entstehung des Bundes-Verfassungsgesetzes
}

\author{
The discussion about the distribution of competences \\ during the creation of the Federal Constitutional Act
}

The question of the distribution of competences was one of the most discussed topics during the creation of the Federal Constitutional Act. Hans Kelsen's drafts were of comparatively little significance in this respect; instead, in the autumn of 1919, Chancellor Renner requested the state offices to name areas of competence which, in their view, should be a matter for the federal state. This resulted in a very long and casuistic catalogue, which was further expanded in the course of the negotiations. The political parties were unable to reach an agreement on the school system in particular; other matters remained controversial to the end as well. Ultimately, the adoption of the Federal Constitution was only possible because the two major parties agreed to postpone the entry into force of the competence provisions until a later, still uncertain date, and to retain the competence provisions of the monarchy until then. With the entry into force of the Federal Constitutional Act on 10 November 1920, Austria was formally transformed into a federal state, but in substantial terms - due to the lack of competence provisions - it was by no means a federal state.

Keywords: competence provisions -Federal Constitutional Act-federalism - Hans KELSEN -

\section{Rückblick auf die Kompetenzverteilung der Monarchie bis 1918}

Fragt man nach dem eigentlich Neuen im Bundes-Verfassungsgesetz vom 1. Oktober 1920, so wird man als eine der ersten Antworten die Einführung des bundesstaatlichen Prinzips hören. Fragt man, worin das Wesen eines Bundesstaates bestehe, so wird man als eine der ersten Antworten die Kompetenzverteilung zwischen Bund und Ländern hören. Und dennoch war die Kompetenzverteilung im B-VG nichts von Grund auf Neues - vielmehr konnten die Väter der Verfassung auch in dieser Frage an einem rechtshistorischen Fundament, den Kompetenzbestimmungen der Verfassung von 1867, anknüpfen. Denn wenn auch die Lehre nicht müde wurde zu betonen, dass Cisleithanien kein Bunhttp://dx.doi.org/10.1553/BRGOE2019-2s271 desstaat gewesen war, ${ }^{1}$ so gab es auch hier ein Zentralparlament und mehrere Landesparlamente mit jeweils eigenen Kompetenzen. So wie später das B-VG, so folgte schon die Kompetenzverteilung in Cisleithanien nicht allgemeinen Grundsätzen, sondern war durch kasuistische Kataloge festgelegt, die im Wesentlichen einander ausschließende Kompetenzen für das Land bzw. für den Gesamtstaat vorsahen; mitunter, etwa im Gemeinderecht, kam dem Gesamtstaat nur eine Grundsatzgesetzgebung zu, woran sich Ausführungsgesetze der Länder anschlossen. Andere Methoden einer Kompetenzverteilung, wie etwa der heute im Europarecht

\footnotetext{
${ }^{1}$ SCHMETTERER, Das kaiserliche Erbe 186. Immerhin sprach KELSEN, Staatsrecht 38, von einer „bundesstaatsähnlichen Teilung der Gesetzgebungskompetenz".
} 
angenommene "Anwendungsvorrang" von unionsrechtlichen Normen, waren dagegen dem cisleithanischen Verfassungsrecht fremd und blieben es auch. ${ }^{2}$

Festzuhalten ist allerdings auch, dass die Kompetenzverteilung der Monarchie, die in zwei verschiedenen gesetzgeberischen Akten - der sog. Februarverfassung 1861 und der sog. Dezemberverfassung 1867 - geschaffen worden war, nur Gesetzgebungskompetenzen regelte, nicht auch eine Kompetenzverteilung von Verwaltungsbehörden. Dies hing freilich mit dem Verwaltungsaufbau der Monarchie zusammen, worauf hier nicht eingegangen $\mathrm{zu}$ werden braucht. $^{3}$

\section{Die Kompetenzverteilung in den ersten Verfassungs- entwürfen 1919}

Im Einzelnen war die verfassungsrechtliche Basis für die Kompetenzverteilung der Monarchie aus legistischer Sicht äußerst mangelhaft und hatte im jungen Verfassungsjuristen Hans Kelsen einen ihrer schärfsten Kritiker gefunden. ${ }^{4}$ Gerade dies aber zeigt, dass dem „Architekten“ des B-VG die Probleme der Kompetenzverteilung in Altösterreich bestens bekannt waren. Fünf Jahre nach Veröffentlichung seines grundlegenden Aufsatzes über „Reichsgesetz und Landesgesetz", im Mai 1919, erhielt er von Staatskanzler Karl Renner "den Auftrag, den Entwurf einer Bundesstaatsverfassung auszuarbeiten“, wobei er sich vornahm, "alles Brauchbare aus der bisherigen Verfassung beizubehalten", sich aber gerade bezüglich des neu einzuführenden bundesstaatlichen Prinzips „an die

\footnotetext{
2 SCHMETTERER, Das kaiserliche Erbe 186.

${ }^{3}$ Siehe dazu die Beiträge von Thomas SIMON und Kamila StAudigl-CieCHOWICZ in diesem Band.

${ }^{4}$ KELSEN, Reichsgesetz und Landesgesetz; vgl. dazu den Beitrag von Christoph SCHMETTERER in diesem Band.
}

schweizerische, aber mehr noch an die neue deutsche Reichsverfassung anzulehnen. ${ }^{\text {"5 }}$

Zwischen Mai und September 1919, während Renner bei den Friedensverhandlungen in St. Germain weilte, arbeitete Kelsen sechs Verfassungsentwürfe aus. ${ }^{6}$ Kelsens erster Verfassungsentwurf, der noch Anfang Juli 1919 fertiggestellt wurde, war dabei der länderfreundlichste Entwurf,7 was Indiz dafür sein könnte, dass es Renner war, der ihn bat, die weiteren Entwürfe stärker zentralistisch $\mathrm{zu}$ gestalten. Im gegebenen Zusammenhang erscheint es jedenfalls wesentlich, dass Kelsen die „Republik Deutschösterreich“ gleich im ersten Artikel als einen „Bund“ von „souveränen Länder[n]“ bezeichnete. Vor diesem Hintergrund wird auch die Formulierung der Kompetenzbestimmungen in diesem ersten Entwurf verständlich. Sie war in lediglich drei, aus heutiger Sicht sehr kurz gehaltenen Artikeln geregelt. In Art. 5 übertrugen die „souveränen Länder Niederösterreich etz. [...] ihre Staatsgewalt" in bestimmten, taxativ aufgezählten Angelegenheiten dem Bund. In Art. 6 wurden einige weitere Angelegenheiten ebenfalls taxativ aufgezählt, hier kam lediglich die Gesetzgebung dem Bund, die Vollziehung hingegen den Ländern zu. Art. 7 enthielt dann die abschließende Bestimmung: „Soferne eine Angelegenheit nicht ausdrücklich durch die Bundesverfassung der Gesetzgebung oder auch der Vollziehung des Bundes übertragen ist, verbleibt sie im Geltungsbereich der souveränen Länder.“ Hier ist schon der heutige Art. 15 Abs. 1 B-VG gut erkennbar. Dagegen ist der vorhin genannte Art. 5 im Kelsen-Entwurf I bemerkenswert, da Kelsen hier nicht bloß von Gesetzgebungs- und Vollziehungskompetenz, sondern von „Staats-

\footnotetext{
${ }^{5}$ KeLSEN, Staatsrecht 160f.; vgl. dazu CADORE, Editorischer Bericht 523; OLECHOWSKI, Kelsen 271.

${ }^{6}$ Diese Entwürfe in synoptischer Art und Weise abgedruckt bei SCHMITZ, Vorentwürfe 114-307, sowie auch bei ERMACORA, Materialien IV, 62-243.

${ }^{7}$ SCHMitZ, Vorentwürfe 49; OLECHOWSKI, Kelsen 278.
} 
gewalt" schlechthin spricht. Es ist dies derselbe Kelsen, der etwas mehr als ein Jahr später darauf drängt, Art. 1 B-VG so zu formulieren, dass nicht „alle Gewalt", sondern „alles Recht“ vom Volk auszugehen habe, ${ }^{8}$ und der später, in seinem Verfassungskommentar, schreibt, dass für „die Sphäre des Rechtes [...] nicht Gewalten, sondern nur Rechtsnormen, die Kompetenzen statuieren, in Betracht" kämen!" ${ }^{\prime \prime 9}$ Dass diese Gewalten oder Kompetenzen nun nicht bloß zwischen verschiedenen Organen, sondern zwischen verschiedenen Gebietskörperschaften aufgeteilt waren, mag aus der Sicht der Reinen Rechtslehre wenig Bedeutung haben; politisch war jedoch die von Kelsen gewählte Terminologie geradezu eine Huldigung der bundesstaatlichen Idee. ${ }^{10}$

Dieser Huldigung jedenfalls war auch das bis heute grundlegende Prinzip geschuldet, wonach die Bundeskompetenzen taxativ aufgezählt waren, während die Kompetenzen der Länder generalklauselartig umschrieben waren. Die Formulierung, dass sie „im Geltungsbereiche der souveränen Länder" verbleiben, suggerierte eine ursprüngliche umfassende Regelungskompetenz der Länder. ${ }^{11}$ Und diese war durchaus weit gezogen, umfasste sie doch u.a. das gesamte Schulrecht, das Gewerberecht, Eisenbahn- und Schiffsverkehr, Bergwesen, Forstwesen und noch einiges mehr. ${ }^{12}$ Dem Bund vollständig übertragen waren dagegen u.a. die Auswärtigen Angelegenheiten und das Militärwesen, Bundes-

\footnotetext{
${ }^{8}$ ERMACORA, Quellen 472.

${ }^{9}$ Kelsen, Froehlich, MERKL, Bundesverfassung 65.

${ }^{10}$ Dies ist umso bemerkenswerter, als Kelsen ja die von Föderalisten heftig bekämpfte - These entwickelt hatte, dass der Staat Deutschösterreich 1918 alleine in Wien gegründet worden sei, die nachfolgenden Beitrittserklärungen der Länder keine juristische Relevanz hätten; vgl. dazu SCHENNACH, Staatsgründung 40; SCHMID, Entstehung 92.

${ }^{11}$ Ebd. 80.

${ }^{12} \mathrm{E}$ contrario-Schluss aus den dem Bund übertragenen Angelegenheiten.
}

finanzen, Zollwesen, Zivil- und Strafjustiz, Kultus und Hochschulwesen; in einigen Bereichen, wie insbesondere im Verwaltungsstrafrecht, im Wasser- und Elektrizitätsrecht sollte der Bund lediglich die Grundsätze regeln können, während die „nähere Durchführung“ dieser Grundsätze den Ländern zukam.

Sowohl die erstaunlich föderalismusfreundliche Terminologie als auch die Föderalismusfreundlichkeit des Meritorischen in der Kompetenzverteilung von Kelsens erstem Entwurf erklären sich wenigstens zum Teil daraus, dass Kelsens „Entwurf I“ nicht der allererste Entwurf war, der überhaupt zum späteren B-VG gemacht wurde; vielmehr hatte die christlichsoziale Partei schon am 14. Mai - zwei Tage nach Renners Abreise nach St. Germain - einen eigenen Verfassungsentwurf für einen „deutschen Bundesfreistaat Österreich“ in der KNV eingebracht. ${ }^{13}$ Damit hatte sie sich als erste politische Partei auch offiziell auf die Umwandlung Österreichs in einen Bundesstaat festgelegt. ${ }^{14}$ Der genannte Entwurf bestimmte in Art. 14 Abs. 1, dass der „Wirkungsbereich der Bundesgewalt [...] alle Angelegenheiten [umfasse], die sich auf Rechte, Pflichten und Interessen beziehen, welche den Ländern des Bundesfreistaates gemeinschaftlich sind." Es war dies allerdings eine bloß programmatische Erklärung, denn Abs. 2 enthielt einen wohl als taxativ zu verstehenden Katalog der Bundeskompetenzen, der übrigens wesentlich weiter als jener Kelsens war, enthielt er doch u.a. eine Kompetenz des Bundes zur Regelung des Vereins- und Versammlungsrechtes oder eine Kompetenz im „Arbeiterrecht“. Es fällt auf, dass manche Materien schlechthin genannt wurden, bei anderen Materien dagegen die Wortfolge "die Regelung des" hinzugefügt wurde, es könnte sein, dass in diesen Fällen nur die Gesetzgebung, nicht auch die Vollziehung Bundes-

${ }^{13} 231$ BlgKNV, auch abgedruckt bei ERMACORA, Quellen 29-42.

${ }^{14}$ WIEDERIN, Diskussion 363. 
sache sein sollte. Art. 15 Abs. 1 des Entwurfes enthielt dann eine Generalklausel zugunsten der Länder (die Identität mit den Ziffern des Art. 15 Abs. 1 B-VG ist natürlich Zufall). Schließlich sah der christlichsoziale Entwurf auch die Möglichkeit vor, dass der Bund Gesetzgebungskompetenzen „fallweise oder auf bestimmte Dauer allen oder einzelnen Ländern überlassen“ könne; in bestimmten Landesangelegenheiten, wie etwa dem unteren und mittleren Erziehungswesen, und auch überall dort, wo Bundesmittel in Anspruch genommen würden, sollte die Landesgesetzgebung nur ",unter Mitwirkung der Bundesregierung" erfolgen - wie diese Mitwirkung aussehen sollte, blieb allerdings offen. ${ }^{15}$

Es kann also durchaus nicht gesagt werden, dass der christlichsoziale Entwurf stärker föderalistisch war als Kelsens erster Entwurf, wohl aber, dass die Regelungstechnik komplizierter und die Textierung weniger klar war. Die Entstehungsgeschichte des christlichsozialen Entwurfes ist unbekannt; von jenen christlichsozialen Abgeordneten, die als Einbringende namentlich genannt wurden, steht Michael Mayr an erster Stelle, weshalb vermutet werden kann, dass er auch der Hauptredaktor des Entwurfes war.

\section{Die Stunde der Staatsämter}

Wie bereits hervorgehoben, waren die meisten Entwürfe, die Kelsen zwischen Juli und September 1919 erstellte, stärker zentralistisch als der erste. Dies betrifft vor allem die Zusammensetzung und Kompetenzen des Bundesrates und auch die - aus der Weimarer Reichsverfassung übernommene - Bestimmung „Bundesrecht bricht Landesrecht", die im Entwurf I noch fehlte und erst in die späteren Entwürfen Eingang fand. ${ }^{16}$ Die Kompetenzverteilung dagegen wur-

\footnotetext{
${ }^{15}$ BerCHTOLD, Verfassungsgeschichte 197.

${ }^{16}$ SCHMITZ, Vorentwürfe 138f. Während Kelsen bei Streichung dieser Regelung noch der Ansicht war,
}

de fast in allen anderen Entwürfen unverändert beibehalten, lediglich Entwurf V, der auch sonst eine Sonderstellung unter Kelsens Verfassungsentwürfen einnimmt, wollte auch die „Grundzüge der Verwaltungsorganisation in den Ländern" dem Bund zuordnen. ${ }^{17}$ Es scheint, dass Renner, der von St. Germain aus in regem Briefkontakt mit Kelsen stand, ${ }^{18}$ hier mit Absicht keine Änderungen von seinem legistischen Berater wünschte, weil er diese Frage auf andere Art und Weise lösen wollte. Unmittelbar nach seiner Rückkehr aus St. Germain und der Genehmigung des Friedensvertrages durch die KNV, am 17. Oktober 1919, wurde die Staatsregierung neu gewählt und das Koalitionsabkommen erneuert. Michael Mayr wurde als Staatssekretär "mit dem bloß persönlichen Aufgabenkreis der Mitarbeit an der Verfassungs- und Verwaltungsreform bis zur Verabschiedung der Verfassungsurkunde" angelobt. ${ }^{19}$

Bereits einen Tag zuvor, am 16. Oktober, hatte Renner ein Schreiben an sämtliche Staatsämter sowie auch an den Staatsrechnungshof und die Statistische Zentralkommission gerichtet, in dem er sie aufforderte, nach einem gewissen, von ihm mitgeschickten Schema an der Formulierung eines Kompetenzkatalogs mitzuwirken. Die einzelnen Behörden sollten jene Materien nennen, „deren Einreihung in die Bundesange-

dass sie sich implizit auch aus anderen Verfassungsbestimmungen ableiten ließe (vgl. OleCHOWSKI, Kelsen 285), geht die heutige Lehre von der Gleichrangigkeit einfacher Bundes- und Landesgesetze aus, die einander daher nach der lex posterior-Regel derogieren können; vgl. ausführlich WIEDERIN, Bundesrecht, bes. 268.

${ }^{17}$ SCHMITZ, Vorentwürfe 131. Entwurf V war jener Entwurf, der sich am stärksten an der Weimarer Reichsverfassung orientierte, während bei den übrigen Entwürfen das Vorbild der österreichischen Dezemberverfassung 1867 deutlicher hervortritt.

18 OLECHOWSKI, Kelsen 272.

${ }^{19}$ Arbeiter-Zeitung Nr. 286 v. 18. 10. 1919, 2; BERCHTOLD, Verfassungsgeschichte 205-207; OLECHOWSKI, Kelsen 283. 
legenheiten bei Wahrung der möglichsten Selbständigkeit der Gliedstaaten im Interesse der Aufrechterhaltung der staatlichen und wirtschaftlichen Lebensfähigkeit des Ganzen unbedingt erforderlich" sei. ${ }^{20}$ Bemerkenswert ist, dass das beigelegte Schema zwei Kataloge vorsah, eines für die Gesetzgebung und eines für die Verwaltung; dies wurde auch in vielen der Antwortschreiben als unzweckmäßig kritisiert.

Diese Antwortschreiben gingen in den nachfolgenden sechs Wochen bei der Staatskanzlei ein und waren sehr unterschiedlich. Kurz waren vor allem das Schreiben des Staatsamts für Äußeres, das sich auf einige wenige Punkte beschränkte, und dasjenige des Staatsamts für Verkehrswesen, das rundheraus erklärte, dass ",alle Angelegenheiten des Verkehrswesens" der Bundesgesetzgebung vorbehalten sein müssten..$^{21}$ Am ausführlichsten war die Stellungnahme des Staatsamtes für Land- und Forstwirtschaft, welches einen extrem ausführlichen Katalog der vom Bund zu regelnden Materien übermittelte, der teilweise sogar über das hinausging, was bis 1918 dem Gesamtstaat zugekommen war, insb., was das Wasserrecht betraf. Dies wurde v.a. mit dem großen Bedeutungsaufschwung dieser Materie in den vorangegangenen Jahren begründet. Das Staatsamt übermittelte darüber hinaus auch Überlegungen zur Organisation der land- und forstwirtschaftlichen Verwaltung und zur Problematik von Rahmengesetzen - bei denen Länder schlecht gezwungen werden könnten, Ausführungsgesetze zu erlassen - und schlug sogar vor, umstrittene Landtagsbeschlüsse einem „Länderausschuss", gebildet aus Vertretern aller Bundesländer, zur Prüfung vorzulegen. ${ }^{22}$

Auch das Antwortschreiben des Staatsamtes für Handel, Gewerbe, Industrie und Bauten war

\footnotetext{
${ }^{20}$ Schreiben der Staatskanzlei an die Staatsämter v. 16. 10. 1919, in: ERMACORA, Materialien III, $4 \mathrm{f}$.

${ }^{21}$ ERMACORA, Materialien III, 27-29 (Verkehrswesen), 110-111 (Äußeres).

22 Ebd. 15-26.
}

sehr umfangreich, verlangte u.a. einige Klarstellungen, wie etwa, dass das "Zivilrechtswesen" auch das Handels- und Wechselrecht umfasse, und begründete die einzelnen Punkte - so wie auch das zuvor genannte Staatsamt - meist damit, dass es sich um eine Materie von besonderer Bedeutung handle (was offenbar schon Grund genug war, sie dem Bund zuzuführen). Beim Punkt "Vermessungswesen“ wurde es sogar als eine „Vergeudung von geistigen Kräften und Geldmitteln“ bezeichnet, „wenn man wirklich daran denken wollte, in jedem Lande die solchen Zwecken dienenden Einrichtungen neu zu schaffen. “ ${ }^{23}$

Renner war bei seiner Aussendung wohl von der Überzeugung ausgegangen, dass die Staatsämter - als Nachfolger der k.k. Ministerien - am ehesten jene praktische Erfahrung besäßen, die für die Erstellung eines Kompetenzkataloges notwendig wären. Dass freilich auf diese Weise ein geradezu kleinlich wirkender, überlanger Katalog von Materien, die dem Bund vorbehalten sein müssten, zustande kam, hätte aber absehbar sein müssen. Basierend auf den Rückmeldungen der Staatsämter entwarf die Staatskanzlei einen Kompetenzkatalog; allein die Beschreibung jener Materien, für die der Bund die ausschließliche Gesetzgebungskompetenz haben sollte, umfasste 841 Worte! (Zum Vergleich: der entsprechende Abschnitt in den Kelsen-Entwürfen hatte lediglich 52 Worte umfasst.) Dazu kam eine Aufzählung jener Materien, wo dem Bund die Grundsatzgesetzgebung und die Verordnungsgebung zustehen sollte, eine beispielhafte Aufzählung der Materien, die der Landesgesetzgebung zufallen würden, eine Aufzählung der Materien, wo die Länder nach Art und Weise der lex Starzyński eine Bedarfskompetenz haben sollten und wo es eine echte konkurrierende Gesetzgebung zwischen Bund und Ländern geben sollte. Daran schloss sich eine Aufzählung

\footnotetext{
${ }^{23}$ Ebd. 154-160.
} 
jener Materien an, in welchen der Bund durch eigene Behörden, in welchen er durch Landesbehörden und in welchen er teils durch Bundes-, teils durch Landesbehörden handeln sollte, und schließlich jener Materien, in denen die Landesverwaltung selbständig agieren konnte. ${ }^{24}$

Das ganze Konvolut wurde am 21. Jänner 1920 an die Staatsämter mit der Bitte um nochmalige Äußerung zurückgesendet, und bis Anfang Februar gingen neue Rückmeldungen ein. ${ }^{25}$ Die Zeit drängte: Die Länder, unzufrieden mit dem bisher schleppenden Vorgang der Verfassungsberatungen, hatten für Anfang 1920 aus eigener Initiative eine Länderkonferenz in Salzburg einberufen. Ein geradezu unglaublicher Affront war es, dass die Staatsregierung zu dieser Konferenz nicht eingeladen war, lediglich an Michael Mayr erging eine Einladung ad personam. ${ }^{26}$ Dieser bemühte sich, bis zu dieser Länderkonferenz bereits einen Verfassungsentwurf zustande $\mathrm{zu}$ bringen, der auch die Zustimmung der Staatsregierung besaß. Letztlich wurde der von ihm auf der Konferenz vorgelegte Entwurf aber als „Privatentwurf Mayr" bezeichnet, weil es sich eben um keinen offiziellen Entwurf der Staatsregierung handelte. ${ }^{27}$

Inhaltlich basierte der „Privatentwurf Mayr ${ }^{\prime 28}$ weitgehend auf den Kelsen-Entwürfen, insbesondere dem Kelsen-Entwurf II; für den $\mathrm{Ab}$ schnitt über die Kompetenzverteilung gilt dies insoweit, als er nicht das schwerfällige Schema Renners übernahm, sondern, ähnlich wie die Kelsen-Entwürfe, die Materien in vier Gruppen unterteilte: Art. 10: Gesetzgebung und Verwal-

\footnotetext{
${ }^{24}$ Legistischer Vorentwurf einer Kompetenzverteilung aufgrund der Stellungnahmen der Staatsämter samt Analysen, in: ebd. 118-139.

${ }^{25}$ Ebd. 140-165.

${ }^{26}$ ERMACORA, Materialien I, 89f.

${ }^{27}$ CADORE, Editorischer Bericht 538; OLECHOWSKI, Kelsen 284.

${ }^{28}$ Abgedruckt bei ERMACORA, Quellen 43-66; SCHMITZ, Vorentwürfe 115-307 (fünfte Spalte); ERMACORA, Materialien IV, 290-412 (erste Spalte).
}

tung Bundessache; Art. 11: Gesetzgebung Bundessache, Vollziehung Landessache; Art. 12: Grundsatzgesetzgebung Bundessache, Ausführungsgesetzgebung und Vollziehung Landessache; Art. 13: Gesetzgebung und Vollziehung Landessache. Inhaltlich differierten diese Artikel jedoch stark von jenen Kelsens und waren weit umfangreicher als diese. Der Staatskanzlei war eine legistische Meisterleistung gelungen: den Wust der Wünsche der einzelnen Staatsämter in ein doch noch halbwegs lesbares Ganzes umgeformt zu haben. Der weitgesteckte Forderungsanspruch des Bundes musste geradezu den entschiedenen Widerspruch jedes noch so moderat föderalistisch gesinnten Ländervertreters hervorrufen, wie die Diskussionen in Salzburg und Linz auch schon bald zeigten.

\section{Die Länderkonferenzen in Salzburg und Linz}

Mit der Salzburger Länderkonferenz (15.-17. Februar 1920) hatten die Länder dem Prozess der Verfassungsentstehung eine neue Wendung gegeben, die Staatsregierung vorübergehend entmachtet und selbst die Initiative ergriffen. Wären die Länder auch im weiteren Verlauf so einig gewesen wie beim Entschluss zur Einberufung einer Länderkonferenz, hätte aus dieser oder einer nachfolgenden Länderkonferenz ein Verfassungstext entstehen können, der vielleicht gar nicht von der KNV, sondern von einer unmittelbar von den Ländern gebildeten Konstituante beschlossen hätte werden können. Aber dem war nicht so. Nicht nur gelang es den Ländern nicht, eine gemeinsame Linie zu finden; es gelang ihnen nicht einmal, dass jedes Land mit einer einzigen Stimme sprach. Vielmehr waren auch die Länder zerrissen zwischen den Parteien, und es traten auch auf den Länderkonferezen weniger die Länder als solche, als vielmehr die Parteigenossen über Landesgrenzen hinweg vereint auf, womit die Salzburger Länderkonfe- 
renz den Charakter eines Parallelparlaments zur KNV in Wien annahm.

Drei Tage dauerte die Salzburger Länderkonferenz, und viel wurde über die Umwandlung Österreichs in einen Bundesstaat debattiert. Doch blieben die Debatten bei allgemeinen Erörterungen stehen, für konkrete Fragen wie etwa die Kompetenzverteilung blieb kaum noch Zeit. Immerhin gaben die Christlichsozialen und die Deutschfreiheitlichen eine Erklärung ab, in der sie die Art der Kompetenzverteilung, wie sie im Privatentwurf Mayr vorgegeben war - also die Gliederung der Materien, nicht den Inhalt der einzelnen Kataloge selbst -, guthießen; die Sozialdemokraten konnten oder wollten sich nicht einmal dazu äußern. ${ }^{29}$

Die Salzburger Länderkonferenz fand vom 20.23. April eine Fortsetzung in Linz, und wieder konnte Mayr zu dieser Konferenz einen Verfassungsentwurf mitbringen, den später so bezeichneten "Linzer Entwurf“. ${ }^{30}$ Der ohnehin schon lange Kompetenzkatalog war - ohne dass Gründe hierzu erkennbar wären - nochmals zugunsten des Bundes erweitert worden. Insbesondere sollte der Bund nunmehr auch bestimmen können, welche Abgaben dem Bund und welche den Ländern zustünden. Auch der Einfluss des Bundes auf das ideologisch so heiß umkämpfte Schulwesen wurde gestärkt. Und sogar Einrichtung, Ausrüstung und Ausbildung der Gendarmerie, also der Wachkörper auf dem „flachen Land“, sollten in Gesetzgebung und Verwaltung nicht Landes-, sondern Bundessache sein.

Dies war freilich noch immer nicht das Maximum dessen, was von einigen Politikern gefordert worden war - so verwies etwa der großdeutsche Delegierte Franz Langoth (1944-1945 Oberbürgermeister von Linz) auf der Linzer

\footnotetext{
${ }^{29}$ ERMACORA, Materialien I, $193 \mathrm{f}$.

30 Abgedruckt bei Kelsen, Froehlich, MerKL, Bundesverfassung 463-506, bei ERMACORA, Quellen 106141 sowie auch bei ERMACORA, Materialien IV, 290412 (zweite Spalte).
}

Landeskonferenz auf einen von seiner eigenen Partei ausgearbeiteten Entwurf, der zumindest in einigen Punkten noch zentralistischer war als der "Linzer Entwurf“ und u.a. fast das gesamte Schulwesen dem Bund zugewiesen hatte. ${ }^{31}$ Dagegen erklärte der Christlichsoziale Otto Ender (Landeshauptmann von Vorarlberg 1918-1934, 1933/34 Hauptredaktor der Verfassung 1934), dass der Entwurf seines Parteifreundes Mayr den ,Föderalismus ertötet" habe und keinesfalls als ein christlichsozialer Entwurf bezeichnet werden könne. Bezugnehmend auf die Finanzhoheit des Bundes meinte er: „Wenn der Mann den Schlüssel zur Geldkasse seiner Frau bedingungslos ausliefert, hat er die männliche Gewalt in seinem Haushalte bedingungslos dahingegeben. Dasselbe ist hier im Staatshaushalte der Fall.“32

Scharf kritisiert wurde von Ender auch, dass nach dem vorliegenden Entwurf die gesamte Wasserrechtsgesetzgebung Bundessache sein sollte, was sogar einen Rückschritt hinter den Zustand der Monarchie darstellen würde, als diese Sache von den Ländern gehandhabt worden war. Es entspann sich zu diesem Punkt eine längere Debatte auf der Linzer Konferenz, nicht zuletzt aus dem Grund, weil der Wasserreichtum der Alpen eine der wenigen Ressourcen darstellte, die der kleinen Republik geblieben waren, und das Potential von (noch $\mathrm{zu}$ errichtenden) Wasserkraftwerken allseits erkannt worden war. Dabei zeigte sich erneut, dass es weniger die Länder waren, die gegensätzliche Meinungen vertraten, sondern dass die Fronten quer durch die Länder gingen: Während der steiermärkische Landeshauptmann Anton Rintelen (1919-1926, 1928-1933, christlichsoziale Par-

\footnotetext{
${ }^{31}$ ERMACORA, Materialien I, 209. Der von Langoth angekündigte Entwurf wurde wenige Tage später in der KNV eingebracht: $842 \mathrm{BlgKNV}$, abgedruckt auch bei ERMACORA, Quellen 78-105, sowie ERMACORA, Materialien IV, 603-627.

32 ERMACORA, Materialien I, 212.
} 
tei) erklärte, dass demjenigen, der die Nachteile des Wassers (= Überflutungen etc.) habe, auch die Vorteile zukommen sollten, und daher das Wasserrecht und das Elektrizitätswesen den Ländern zustehen müssten, war sein sozialdemokratischer Stellvertreter Josef Pongratz der Meinung, dass „es sich hier um große wirtschaftliche Fragen handle, die in der Regel mehrere Länder berühren", weshalb eine Kompetenz des Bundes zu befürworten sei. ${ }^{33}$

So ging auch die Linzer Konferenz zu Ende, ohne dass ein Kompromiss erzielt werden hatte können. Immerhin beschlossen Renner und Mayr sowie auch Vizekanzler Jodok Fink, unter Mitwirkung Kelsens und auf Grundlage der bisher vorliegenden Entwürfe einen gemeinsamen Entwurf zustande zu bringen; auch dies scheiterte allerdings. ${ }^{34}$ Nach dem Bruch der Großen Koalition am 11. Juni, und nachdem die christlichsoziale Partei unter Mayr am 25. Juni einen neuen Verfassungsentwurf in der KNV eingebracht hatte, ${ }^{35}$ veröffentlichte Renner das Ergebnis der Beratungen, die er mit Mayr, Fink und Kelsen geführt hatte, in der "Wiener Zeitung" (sog. Renner-Mayr-Entwurf). ${ }^{36}$ Dabei erwies es sich, dass über Struktur und Aufbau der Verfassung Einigkeit bestand, und ebenso über eine Reihe anderer wichtiger, inhaltlicher Punkte. Nur bei wenigen, aber entscheidenden Themen hatte es keinen Kompromiss gegeben; in der „Wiener Zeitung“ wurden diesfalls die beiden Varianten nacheinander abgedruckt, so insbesondere in der Frage der Kompetenzverteilung. Hervorzuheben ist am Renner-Mayr-Entwurf, dass die Kompetenztatbestände innerhalb der jeweiligen Artikel so angeordnet waren, dass sie

\footnotetext{
${ }^{33}$ Offizieller Bericht des Pressekomitees über die vertraulichen Beratungen am 22.4. 1920, in: ERMACORA, Materialien III, 195.

${ }^{34}$ CADORE, Editorischer Bericht 545; OlECHOWSKI, Kelsen 287.

35888 BlgKNV, abgedruckt auch bei ERMACORA, Quellen 141-151.

${ }^{36}$ ERMACORA, Quellen 188-268.
}

auch als "Grundlage der Geschäftseinteilung" der Bundesministerien (wie sie heute im Bundesministeriengesetz geregelt ist) dienen konnte. So wurden „im Punkt 1 die Geschäfte der Bundeskanzlei, im Punkt 2 die des Ministeriums für Äußeres, 3 für Heerwesen" usw. aufgezählt. ${ }^{37}$ Diese Idee wurde später vom Verfassungsunterausschuss wieder aufgegeben, weil die Zuordnung der Materien $\mathrm{zu}$ Ministerien doch stets Schwankungen unterworfen sein können sollte, vor allem aber, weil es auch Materien gab, die keinem Ministerium eindeutig zugeordnet werden konnten, wie z.B. die Verwaltungsgerichtsbarkeit. ${ }^{38}$

\section{In der Konstituierenden Nationalversammlung}

Mit dem Bruch der Koalition war das Zustandekommen der Bundesverfassung scheinbar in weite Ferne gerückt. Neuwahlen der KNV wurden für den 17. Oktober ausgeschrieben, bis dahin sollte eine Proporzregierung aus allen im Parlament vertretenen Parteien unter Vorsitz Mayrs die Geschäfte weiterführen; von hier sollte keine Initiative mehr kommen. ${ }^{39}$

Doch gerade in dieser Situation wurde die KNV, also das eigentlich für den Verfassungsbeschluss zuständige Organ, das bislang in dieser Hinsicht untätig geblieben war, aktiv. Der Vorsitzende des Verfassungsausschusses, der Sozialdemokrat Otto Bauer, und sein Stellvertreter, der Christlichsoziale Ignaz Seipel, vereinbarten, noch vor den Wahlen einen Kompromiss zustande bringen $\mathrm{zu}$ wollen. Ein Unterausschuss wurde gebildet, der am 11. Juli seine Beratungen aufnahm. Als Tischvorlage diente der „Linzer

\footnotetext{
37 ERMACORA, Quellen 257.

${ }^{38}$ ERMACORA, Quellen 269.

${ }^{39}$ CADORE, Editorischer Bericht 547f.; OlECHOWSKI, Kelsen 287f.
} 
Entwurf ${ }^{\prime \prime}, 40$ doch wurde immer wieder auch auf andere Entwürfe verwiesen.

Der Unterausschuss wandte sich gleich zu Beginn seiner Beratungen dem Problem der Kompetenzabgrenzung $\mathrm{zu}$, und die Beratungen gingen zunächst rasch voran: Punkt für Punkt wurde der Linzer Entwurf durchberaten, und für eine Reihe von Änderungen gab es Zustimmung, häufig jedoch nur mit Stimmenmehrheit, sodass zu befürchten war, dass der endgültige Text die Zweidrittelmehrheit in der KNV verfehlen würde.

Oftmals führten die Diskussionen auch zu Auseinandersetzungen um meritorische Fragen der im Kompetenzkatalog aufgezählten Regelungsgegenstände, so etwa, ob es zweckmäßig sei, permanente Wählerlisten („Bürgerlisten“) einzuführen. ${ }^{41}$ Eng mit der Kompetenzverteilung in Zusammenhang stehend war die Frage, ob jene Materien, zu deren Vollzug der Bund zuständig sein sollte, auch wirklich von Bundesbehörden zu vollziehen seien, oder ob nicht Landesbehör-

\footnotetext{
${ }^{40}$ ERMACORA, Quellen 270. In der Literatur war es lange strittig, ob mit „Linzer Entwurf“ der von Michael Mayr zur Linzer Länderkonferenz gebrachte Entwurf (so WALTER, THIENEL, Parlament 22) oder das Ergebnis dieser Verhandlungen (so ERMACORA, Quellen 106) gemeint sei. Eine genaue Textanalyse sowie auch das nach der Sitzung vom 22. 7. ausgegebene Communiqué sprechen eindeutig für die erstgenannte Ansicht. Dies ist auch leicht nachzuvollziehen, zumal der zur Länderkonferenz eingebrachte Entwurf von der Österreichischen Staatsdruckerei gedruckt und daher wohl in großer Zahl vorhanden war, während die Ergebnisse der Verhandlungen keinen zeitgenössischen Druck erfuhren; die Edition bei ERMACORA basiert offenbar auf einem im ÖStA, AdR, Büro Seitz, Karton 7, vorhandenen Original, wo die auf der Linzer Konferenz beschlossenen Änderungen mit einer roten Schreibmaschinenschrift im Druck ersichtlich gemacht wurde, weshalb die Vermutung naheliegt, dass es sich um ein Unikat handelte.

${ }^{41}$ Derartige Listen wurden erst mit der Nationalratswahlordnung vom 11. 7. 1923 BGB1 367 eingeführt, bis dahin wurden für jede Wahl neue Listen erstellt, was dementsprechend viele Probleme bedeutete, vgl. dazu KELSEN, Wählerlisten 290; STREJCEK, Entwicklung 47.
}

den - im Rahmen der sog. mittelbaren Bundesverwaltung - hier für den Bund tätig sein sollten. Der Linzer Entwurf (Art. 92) hatte die mittelbare Bundesverwaltung sogar zum Regelfall erklärt, von dem aber durch Bundesgesetz Ausnahmen gemacht werden konnten. Demgegenüber hatte der zweite christlichsoziale Entwurf eine eigene Aufzählung vorgesehen, für welche Angelegenheiten eigene Bundesbehörden geschaffen werden könnten. ${ }^{42}$ Darauf wurde nunmehr bei den Beratungen im Verfassungsunterausschuss verwiesen, ${ }^{43}$ worauf die Staatskanzlei drei Wochen später einen Textvorschlag in die Verhandlungen einbrachte, der sich zwar inhaltlich am christlichsozialen Vorschlag orientierte, aber die Formulierung der einzelnen Materien an jene der Kompetenzverteilung anglich und auch inhaltlich weit über die ursprüngliche Aufzählung hinausging; so zählten jetzt auch u.a. das Schul- und Erziehungswesen sowie der Denkmalschutz zu jenen Materien, in denen der Bund durch eigene Behörden handeln konnte. Obwohl der Staatskanzlei nur wenig Zeit zur Verfügung gestanden war, hatte sie ihre Textierung unter nochmaliger Einbindung der Staatsämter erstellt; der Leiter des Verfassungsdienstes der Staatskanzlei, Georg Froehlich, erklärte, dass die Arbeit „außerordentliche Schwierigkeiten" bereitet habe, da es sich bei vielen Ämtern in Wirklichkeit um „Wirtschaftsstellen der staatlichen Privatwirtschaft" handle. Einzelne Formulierungen wurden im Verfassungsunterausschuss noch beanstandet und abgeändert, so etwa auf Antrag Seipels der Terminus „Justizpflege" in "Justizwesen“. Dennoch konnte hier relativ rasch eine Einigung erzielt werden. ${ }^{44}$

Am selben Tag, dem 14. September, tagte der christlichsoziale Parteivorstand und zog eine

${ }^{42}$ Zweiter Entwurf der Christlichsozialen, 888 BlgKNV, Art. 26, abgedruckt bei ERMACORA, Quellen 149.

${ }^{43}$ ERMACORA, Quellen 331.

${ }^{44}$ Ebd. 438f. 
Reihe von roten Linien für die Verfassungsberatungen. Seipel berichtete am folgenden Tag im Verfassungsunterausschuss, dass die christlichsoziale Partei „einer Kompetenzabgrenzung, die das gesamte Schulwesen einschließlich der Volks- und Bürgerschulen der Kompetenz des Bundes zuweist, nicht zustimmen [könne]." Insbesondere das Dienstrecht der Lehrer an Volksund Bürgerschulen sowie auch die Schulaufsicht müssten Landessache bleiben, wobei Seipel bei letzterem einen Kompromissvorschlag präsentierte. Der zweite Punkt, den die christlichsoziale Partei dezidiert ablehnte, war die Einführung von Kreisgemeinden. ${ }^{45}$

\section{Der Kompromiss}

Damit standen die gesamten Verfassungsberatungen wieder einmal knapp vor dem Scheitern. In diesem entscheidenden Moment kam es, am 18. September, $\mathrm{zu}$ einer Parteienübereinkunft auf höchster Ebene zwischen Sozialdemokraten und Christlichsozialen: Alles, worüber ein Kompromiss hatte erzielt werden können, sollte von der KNV als Bundes-Verfassungsgesetz beschlossen werden; überall dort, wo dieser Kompromiss fehlte, sollte der Rechtszustand der Monarchie vorläufig beibehalten werden. ${ }^{46}$

Unter diesem gewichtigen Vorbehalt passierte der Verfassungstext am 23. September den Unterausschuss, am 25. September den Verfassungsausschuss und am 1. Oktober das Plenum der KNV. Dieser Verfassungstext regelte in seinen Art. 10-15 die Kompetenzverteilung zwischen Bund und Ländern, wobei jedoch schon

\footnotetext{
${ }^{45}$ ERMACORA, Quellen 446.

${ }^{46}$ Es ist bezeichnend, dass unter den tausenden Seiten an Quellen zur Verfassungsentstehung ausgerechnet für diesen so entscheidenden Kompromiss keine direkten schriftlichen Aufzeichnungen bekannt sind, sondern uns der Inhalt nur durch Zeitungsberichte bekannt ist: Arbeiter-Zeitung Nr. 259 v. 19. 9. 1920, 1; Neue Freie Presse Nr. 20138 v. 19. 9. 1920, 6.
}

Art. 14 festhielt, dass „[a]uf dem Gebiet des Schul-, Erziehungs- und Volksbildungswesens [...] der Wirkungsbereich des Bundes und der Länder durch ein besonderes Bundesverfassungsgesetz geregelt" werden würde. Vor allem aber wurde parallel zum B-VG ein VerfassungsÜbergangsgesetz beschlossen, das in $\S 42$ festhielt, dass die gesamte Kompetenzverteilung erst dann in Kraft treten solle, wenn auch dieses Schul-Verfassungsgesetz in Kraft trete, außerdem aber auch noch ein Verfassungsgesetz über die finanzielle Auseinandersetzung zwischen Bund, Ländern und Gemeinden sowie ein Verfassungsgesetz, das die Organisation der allgemeinen staatlichen Verwaltung in den Ländern regeln würde. Bis dahin sollten die „Zuständigkeiten zwischen Bund und Ländern" in Gesetzgebung und Verwaltung "gegenüber der bestehenden zwischen Staat und Ländern" nicht geändert werden.

Damit wurde für eine ungewisse Übergangszeit eine ausgesprochen unklare Rechtslage perpetuiert und noch weiter verkompliziert. Der Umfang der Landesgesetzgebung richtete sich im Wesentlichen weiterhin nach den $\S \S 18$ f. der praktisch gleichlautenden - Landesordnungen von 1861; nicht alles jedoch durfte auch von den Ländern selbst vollzogen werden, sodass es nunmehr auch dazu kommen konnte, dass manche Angelegenheiten in Gesetzgebung Landessache, in der Vollziehung Bundessache waren ${ }^{47}$ wobei der Umstand, dass der Großteil der Bundesverwaltung in der Form der mittelbaren Bundesverwaltung erfolgte, diesen bedeutsamen Unterschied nochmals verschleierte und undurchsichtig machte. Dass ein Rechtsirrtum eines Verwaltungsorgans angesichts dieser Situation durchaus „entschuldbar" sein konnte, musste 1923 selbst der Verfassungsgerichtshof zugeben. In diesem spektakulären Fall hatte der (sozialdemokratische) Landeshauptmann von

\footnotetext{
${ }^{47}$ BERCHTOLD, Verfassungsgeschichte 377.
} 
Wien, gestützt auf eine Bestimmung des Reichssanitätsgesetzes $1879^{48}$ und entgegen einer Weisung des (christlichsozialen) Innenministers, die Bewilligung zur Inbetriebnahme eines Krematoriums in Wien-Simmering erteilt. Nach Art. 10 Abs. 12 B-VG waren das „Leichen- und Bestattungswesen“ ausdrücklich von der Kompetenz des Bundes in Gesetzgebung und Vollziehung ausgenommen, doch war die fragliche Bestimmung gem. § 42 Verfassungs-Übergangsgesetz noch nicht in Kraft; vielmehr handelte es sich um eine Materie, die in mittelbarer Bundesverwaltung zu vollziehen gewesen wäre. Die Weisung des Innenministers wäre also durchaus beachtlich gewesen, doch angesichts der verworrenen Rechtslage wollte der VfGH dem Landeshauptmann keinen Vorwurf machen. ${ }^{49}$

In diesem Fall war das verfassungsgerichtliche Erkenntnis zur Zufriedenheit der Sozialdemokraten ausgefallen. Wenige Monate später jedoch, im Juni 1924, hob der VfGH auf Antrag der (christlichsozialen) Salzburger Landesregierung einen Großteil der 7. Krankenversicherungsnovelle wegen Unzuständigkeit des Bundesgesetzgebers auf; abermals waren es die komplizierten Übergangsregelungen, die den Ausschlag für dieses Erkenntnis gegeben hatten. ${ }^{50}$ Nun erkannte auch Otto Bauer, dass der derzeitige Zustand der Kompetenzverteilung „unerträglich“ sei. ${ }^{51}$

Dies erleichterte sicherlich ein Jahr später das Zustandekommen der Verfassungsnovelle 1925, mit der u.a. der $\S 42$ Verfassungs-Übergangsgesetz völlig neu gefasst wurde, sodass die vom B-VG vorgezeichnete Kompetenzverteilung

\footnotetext{
${ }^{48}$ Gesetz 30. 4. 1870 RGBl. 68 betreffend die Organisation des öffentlichen Sanitätsdienstes.

${ }^{49} \mathrm{VfGH}$ 27.3. 1923 VfSlg 206. Dass die politischen Hintergründe des Falles mindestens ebenso verwickelt waren wie die juristischen Probleme, braucht hier nicht hervorgehoben werden, vgl. dazu schon OlECHOWSKI, Kelsen $433 \mathrm{f}$.

${ }^{50}$ VfGH 27. 6. 1924 VfSlg 328.

${ }^{51}$ BERCHTOLD, Verfassungsgeschichte 377.
}

zwischen Bund und Ländern - außer im Bereich des Schulwesens und mit einigen Änderungen gegenüber dem Kompetenzkatalog von 1920 mit 1. Oktober 1925 in Kraft treten konnte. ${ }^{52}$ Das im „alten“ § 42 angekündigte Finanz-Verfassungsgesetz hatte schon 1922 erlassen werden können. ${ }^{53}$ Lediglich in der ideologisch so heiklen Schulfrage gelang eine Lösung erst 1962, in der Zweiten Republik. ${ }^{54}$

\section{Resümee}

Der Wortlaut der Art. 10-15 B-VG, der in überaus kasuistischer Weise die Zuständigkeiten des Bundes und der Länder zu Gesetzgebung und Vollziehung regelt, hatte innerhalb der Verfassungsentstehung eine durchaus eigenständige Genese. Er stammt nicht von Kelsen, und es ist auch sonst nicht das Werk eines großen Staatstheoretikers darin zu erblicken. Vielmehr kamen die Tatbestände direkt aus der Verwaltungspraxis. Konkret waren es die einzelnen Staatsämter (die Nachfolger der k.k. Ministerien und Vorläufer der heutigen Bundesministerien), die hier einfach auflisteten, womit sie im Alltag zu tun hatten bzw. was sie für unentbehrlich für ihren Wirkungskreis hielten, und waren es verschiedenste Interessenvertreter aller politischen Parteien, die in zermürbender Kleinarbeit noch diese und jene Änderung um jeden Preis - auch um den Preis eines möglichen Scheiterns der gesamten Verfassung - erzwingen wollten.

Der VfGH hat bereits in der Ersten Republik die Theorie entwickelt, dass ein vom B-VG verwendeter Begriff auch heute noch jene Bedeutung habe, die er zu jenem Zeitpunkt gehabt habe, zu dem die betreffende Bestimmung in Kraft getre-

\footnotetext{
52 Art. III BVG 30. 7. 1925 BGBl. 269 (Übergangsnovelle). 53 BVG 3. 3. 1922 BGBl. 124 (Finanz-Verfassungsgesetz); vgl. zu seiner Genese BERCHTOLD, Verfassungsgeschichte 330-335.

${ }^{54}$ BVG 18. 7. 1962 BGBl. 215 (Schulverfassungsnovelle); vgl. zu ihr WALter, Thienel, Parlament 79-81.
} 
ten sei; die damalige Bedeutung aber sei mit Blick auf den damaligen Stand der Gesetzgebung zu ermitteln. ${ }^{55}$ Diese sog. Versteinerungstheorie hat zeitweise eine außerordentliche Bedeutung für die Verfassungsinterpretation erlangt, ihren Höhepunkt aber schon seit langem überschritten; für die Interpretation der Kompetenztatbestände der Art. 10-15 B-VG wird ihre Brauchbarkeit aber nach wie vor anerkannt. Fraglich ist, ob es sich bei dieser - etwas unzweckmäßig als „Theorie“ bezeichneten - Interpretationsmethode um eine historische Interpretation handelt, was z.T. bezweifelt wird. ${ }^{56}$ Die vorstehenden Ausführungen haben gezeigt, dass eine Person, die heutzutage die einfachgesetzliche Lage zum Zeitpunkt des Inkrafttretens der Kompetenzbestimmungen untersucht, im Grunde nichts Anderes macht als die Staatsämter und Lobbyisten von damals und damit tatsächlich den Willen des Verfassungsgesetzebers empirisch erforscht. ${ }^{57}$ Somit kann die Versteinerungsmethode im Prinzip sehr wohl als eine „historische Interpretation" angesehen werden; freilich würde es sich bei dieser Methode empfehlen, nicht nur die Gesetze jener Zeit, sondern insbesondere auch die oben angeführten Schreiben der Staatsämter zur Ermittlung dieses „wahren Willens" heranzuziehen.

${ }^{55}$ Grundlegend SCHÄFFER, Verfassungsinterpretation 97-116.

${ }^{56}$ Siehe die Übersicht der Lehrmeinungen bei WIEDERIN, Anmerkungen 1233.

${ }^{57}$ Damit sei insbesondere dem Einwand von WIEDERIN, Anmerkungen 1236, begegnet, es werde der „Wille des Verfassungsgesetzgebers [...] nicht empirisch erforscht." Natürlich kann darauf erwidert werden, dass der tatsächliche Wille der Bürokraten und Lobbyisten nicht mit dem tatsächlichen Willen der Abgeordneten übereinstimmen muss - doch ist dies ein allgemeines Problem der historischen Interpretation; vgl. JABLONER, Gesetzesmaterialien 65.

\section{Korrespondenz:}

Prof. Dr. Thomas OleCHOWSKI

Universität Wien

Institut für Rechts- und Verfassungsgeschichte

Schottenbastei 10-16

1010 Wien

thomas.olechowski@univie.ac.at

ORCID-Nr. 0000-0003-3291-6876

\section{Abkürzungen:}

AdR Archiv der Republik

BlgKNV Beilagen zu den Stenographischen Protokollen der Konstituierenden Nationalversammlung

ÖStA Österreichisches Staatsarchiv

VfSlg Sammlung der Erkenntnisse und wichtigsten Beschlüsse des Verfassungsgerichtshofes

Siehe auch das allgemeine Abkürzungsverzeichnis: [http://www.rechtsgeschichte.at/media/abk.pdf]

\section{Literatur:}

Klaus BERCHTOLD, Verfassungsgeschichte der Republik Österreich I: 1918-1933 [einziger Band] (Wien 1998).

Rodrigo CADORE, Editorischer Bericht, in: Matthias JESTAEDT (Hg.), Hans Kelsen Werke, Bd. VIII (Tübingen 2020) 505-573.

Felix ERMACORA, Quellen zum Österreichischen Verfassungsrecht (1920) (= Mitteilungen des Österreichischen Staatsarchivs, ErgBd. VIII, Wien 1967).

DERS., Materialien zur Österreichischen Bundesverfassung I: Die Länderkonferenzen 1919/20 und die Verfassungsfrage (= Österreichische Schriftenreihe für Rechts- und Politikwissenschaft IX/1, Wien 1989).

DERS., Materialien zur Österreichischen Bundesverfassung III: Die Aufgabenverteilung zwischen Bund und Ländern (= Österreichische Schriftenreihe für Rechts- und Politikwissenschaft IX/3, Wien 1986).

DERS., Materialien zur Österreichischen Bundesverfassung IV: Die Sammlung der Entwürfe zur Staats- bzw. Bundesverfassung (= Österreichische Schriftenreihe für Rechts- und Politikwissenschaft IX/4, Wien 1990).

Clemens JABLONER, Die Gesetzesmaterialien als Mittel der historischen Auslegung (1994), Neudruck in: 
Clemens JABLONER, Methodenreinheit und Erkenntnisvielfalt. Aufsätze zur Rechtstheorie, Rechtsdogmatik und Rechtsgeschichte (= Schriftenreihe des Hans Kelsen-Instituts 35, Wien 2013) $61-77$.

Hans KELSEN, Wählerlisten und Reklamationsrecht, in: JBl 35 (1906) 289-290, 301-304, 316-318, 327329; Neudruck in: Matthias JestAedt (Hg.), Hans Kelsen Werke, Bd. I (Tübingen 2007) 301-331.

DERS., Reichsgesetz und Landesgesetz nach österreichischer Verfassung, in: AöR 32 (1914) 202-245, 390-438; Neudruck in: Matthias JestaedT (Hg.), Hans Kelsen Werke, Bd. III (Tübingen 2010) 359425.

Ders., Georg Froehlich, Adolf Merkl (Hgg.), Die Verfassungsgesetze der Republik Österreich, Bd. V: Die Bundesverfassung vom 1. Oktober 1920 (Wien-Leipzig 1922), Neudruck in: Matthias JESTAEDT (Hg.), Hans Kelsen Werke, Bd. VIII (Tübingen 2020) 25-502.

DERS., Österreichisches Staatsrecht. Ein Grundriß entwicklungsgeschichtlich dargestellt (Tübingen 1923, ND Aalen 1981).

Thomas OLECHOWSKI, Hans Kelsen. Biographie eines Rechtswissenschaftlers (Tübingen 22021).

Heinz SCHÄFFER, Verfassungsinterpretation in Österreich. Eine kritische Bestandaufnahme (= Forschungen aus Staat und Recht 18, Wien-New York 1971).

Martin P. SCHENNACH, Die Staatsgründung 1918 und die Länder, in: Peter BußjÄGER (Hg.), 3. November 1918 - Die Länder und der neue Staat (WienHamburg 2019) 39-56.
Christoph SCHMETTERER, Das kaiserliche Erbe im B-VG, in: ZÖR 75 (2020) 175-191.

Sebastian SCHMID, Die Entstehung der Republik aus Sicht der Länder, in: Clemens JABLONER u.a. (Hgg.) Die Verfassungsentwicklung 1918-1920 und Hans Kelsen (= Schriftenreihe des Hans Kelsen-Instituts 41, Wien 2020) 85-100.

Georg SCHMITZ, Die Vorentwürfe Hans Kelsens für die österreichische Bundesverfassung (= Schriftenreihe des Hans Kelsen-Instituts 6, Wien 1981).

Gerhard STREJCEK, Zur Entwicklung der Wahlgrundsätze und der Wahlprüfung. Ein Beitrag zur Analyse der Beck'schen Wahlreform anlässlich der Hundertjahrfeier, in: Thomas SIMON (Hg.), Hundert Jahre allgemeines und gleiches Wahlrecht in Österreich (= Rechtshistorische Reihe 400, Frankfurt a.M. u.a. 2010) 37-51.

Robert WALTER, Rudolf THIENEL, Parlament und Bundesverfassung. Der Beitrag des Parlaments zur Entstehung und Entwicklung des Bundes-Verfassungsgesetzes (= Juristische Schriftenreihe 21, Wien 1990).

Ewald WIEDERIN, Bundesrecht und Landesrecht (=Forschungen aus Staat und Recht 111, WienNew York 1995).

DERS., Anmerkungen zur Versteinerungstheorie, in: Herbert HALLER u.a. (Hgg.), Staat und Recht. Festschrift für Günther Winkler (Wien-New York 1997) 1231-1272.

DERS., Die verfassungspolitische Diskussion über die Einrichtung Österreichs als Bundesstaat, in: BRGÖ 1 (2011) 356-373. 